\title{
Efficient preparation of graphene liquid cell utilizing direct transfer with large-area well-stitched graphene
}

\author{
Yuki Sasaki $^{\mathrm{a}}$, Ryo Kitaura ${ }^{\mathrm{a}}$, Jong Min Yuk ${ }^{\mathrm{b}, \mathrm{c}, \mathrm{d}},{\text { Alex } \text { Zettl }^{\mathrm{b}, \mathrm{c}, \mathrm{d}} \text { and Hisanori Shinohara }}^{\mathrm{a} *}$ \\ ${ }^{a}$ Department of Chemistry \& Institute for Advanced Research, Nagoya University, \\ Nagoya 464-8602, Japan. \\ ${ }^{b}$ Department of Physics, University of California at Berkeley, Berkeley, California 94720 , \\ USA. \\ ${ }^{c}$ Materials Sciences Division, Lawrence Berkeley National Laboratory, Berkeley, \\ California 94720, USA. \\ ${ }^{d}$ Kavli Energy NanoScience Institute at the University of California, Berkeley and the \\ Lawrence Berkeley National Laboratory, Berkeley, California 94720, USA. \\ E-mail: r.kitaura@nagoya-u.jp,noris@nagoya-u.jp
}




\begin{abstract}
By utilizing graphene-sandwiched structures recently developed in this laboratory, we are able to visualize small droplets of liquids in nanometer scale. We have found that small water droplets as small as several tens of nanometers sandwiched by two single-layer graphene are frequently observed by TEM. Due to the electron beam irradiation during the TEM observation, these sandwiched droplets are frequently moving from one place to another and are subjected to create small bubbles inside. The synthesis of a large area single-domain graphene of high-quality is essential to prepare the graphene sandwiched cell which safely encapsulates the droplets in nanometer size.
\end{abstract}

\title{
Graphical abstract
}

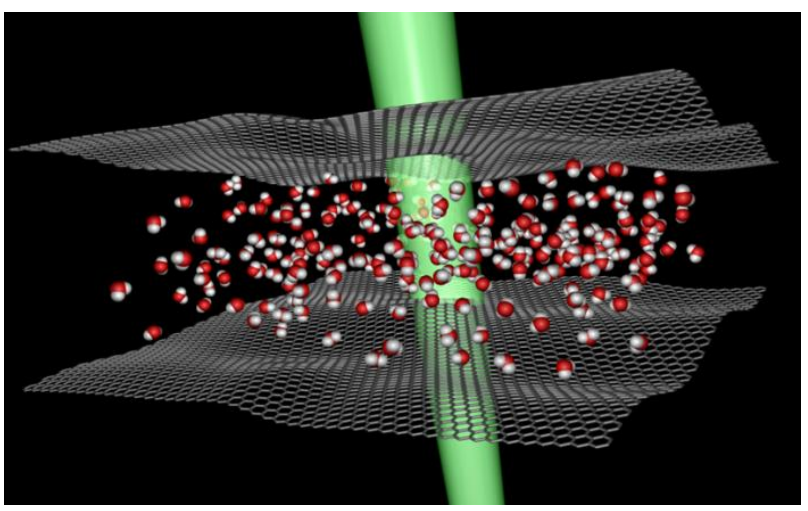




\section{Introduction}

Electron microscope observation of solution and solution-based processes is one of the important issues in nanometer-scale science. For example, the direct structural observation of biomolecules, such as proteins, in water provides crucial information to understand how the protein works in living systems[1-4] and how physical changes occur in solution such as dissolution, precipitation and dispersion[5-7]. Until now, great research efforts have been devoted to elucidate the microscopic structure and dynamics of solution, in which various spectroscopic techniques have been widely employed to obtain information on various phenomena in solutions[8-11].

Observation of solutions using transmission electron microscopy (TEM) can, in principle, provides direct information on microstructure of solution at atomic level[12-18]. Recent advancement on TEM has enabled us to observe structure of matters with spatial and time resolution down to $0.05 \mathrm{~nm}$ and 1 millisecond, respectively[19, 20]. In addition, development of low-acceleration voltage technique has greatly expanded versatility of TEM, enabling us to observe bio-organic fragile materials at atomic resolution[21]. High-resolution TEM simultaneously gives us information on electronic structure and bonding state[22]. If TEM can equally be applied to observe solution, it must be one of the most powerful characterization techniques to provide direct and high-resolution structural information on solution.

TEM observation of solutions have achieved by using environmental cell[23, 24]. Recently we demonstrate graphene liquid cells: liquids sandwiched by two single-layer graphene sheets for visualization of metal particles in solution with atomic resolution $[16,17,25]$. Graphene is the one-atom-thick carbon sheet, and due to the thinnest 
structure composed of a light element, graphene is almost transparent to electron beam[26, 27]. In addition, graphene is a mechanically and chemically stable conductor [28-30], and high-contrast and stable TEM observation can be done using graphene as a sample support [31-33]. In addition, even a single-layer graphene has the perfect impermeability, and if various solutions can be sandwiched between two graphene layers, the solution can be supported stably even under an UHV condition, which provides TEM observations of microstructure of solutions $[17,34]$.

There are, however, some unsavory points corresponding to reproducibility and yield of graphene liquid cells because of vulnerability of low-crystalline graphene sheets. It is important to utilize high-quality graphene to ease the fabrication of the graphene liquid cells as well as of graphene nano-ribbons [34-36].

Here, we have development a simple technique for preparation of the graphene liquid cell by utilizing direct transfer process with large-area and high-quality graphene grown on copper. The large area high-quality graphene was based on the chemical vapor deposition (CVD) on copper foils, which has been developed by Ruoff and coworkers $[37,38]$. The pretreatment of copper foils with atmospheric pressure of pure hydrogen gas provides well-stitched and large-area graphene within a relatively short reaction time. By using such a well-stitched graphene and direct transfer process, we are able to prepare the graphene liquid cell in high yield.

\section{Materials and methods}

We used copper foils as substrates for graphene growth. A copper foil (nilaco, 99.9 $\%, 10 \times 100 \times 0.02 \mathrm{~mm}$ ) was placed in a quartz tube and annealed at $1323 \mathrm{~K}$ under 500 
sccm flow of $100 \%$ hydrogen for 50 minutes [37, 39, 40]. After the pre-anneal treatment, copper (111) surface preferentially appears. Graphene growth was then performed under $250 \mathrm{sccm}$ flow of $\mathrm{Ar} / \mathrm{H}_{2} / \mathrm{CH}_{4}\left(\mathrm{Ar}: 97 \%, \mathrm{H}_{2}: 3 \%, \mathrm{CH}_{4}: 0.0002 \%\right.$ ) at the same temperature. The typical growth time of a $\mathrm{mm}$ size graphene is $8 \sim 16$ hours.

Graphene grown on a copper foil was transferred onto a TEM grid (SiN membrane film with 2 um pore) using the direct transfer method. To remove copper substrates, a copper foil with a continuous film of graphene grown on the surface was placed on aqueous solution of ammonium peroxodisulfate $(1 \mathrm{~mol} / \mathrm{L})$ for 12 hours (see supplementary information). This process can remove the copper foil almost completely, leading to the graphene film floating on the etching solution, the graphene film was picked up by a SiN TEM grid, and the picked-up graphene was then carefully placed onto aqueous solution of hydrochloric acid to remove copper residues. Before fabrication of a graphene liquid cell, the so-washed graphene on the TEM grid was further cleaned with hydrogen plasma treatment[31, 41].

The TEM observation was made with either a field-emission type JEM-2100F or JEM-2010 with an acceleration voltage of $80 \mathrm{kV}$. Typical exposure time and current density were $0.3 \mathrm{~s}$ and $96 \mathrm{~A} / \mathrm{cm}^{2}$, respectively.

\section{Results and discussion}

Our strategy to prepare the graphene liquid cell is a simple and direct preparation of a sandwiched structure using a high-quality large-area CVD-grown graphene. Figure 1 shows the preparation scheme of the graphene liquid cell. First, a CVD-grown graphene is transferred onto a $\mathrm{SiN}$ membrane grid with 2 um holes penetrating through the 
membrane. Second, using a mist generator, various solutions are then sprayed onto another graphene specimen floating on etching solution. Third, to form a graphene sandwiched liquid cell, the deposited micro-size droplets of solutions are covered by the originally-transferred graphene on TEM grid. In the preparation of the graphene liquid cell, incorporation of large-area and seamless graphene layers is essential to realize successful encapsulation of solutions. To achieve high-yield preparation of the graphene liquid cell, we have developed a growth technique for high-quality large-area graphene.

Graphene has been synthesized with chemical vapor deposition (CVD) using methane and a cupper foil as the carbon source and the substrate, respectively. The CVD growth of graphene is strongly influenced by the substrate, and (111) plane of cupper has been known as the suitable surface to grow high-quality graphene [42, 43]. To prepare the (111) plane, we have utilized high-temperature pretreatment of cupper foil under pure $\mathrm{H}_{2}$ flow. After the pretreatment, almost all surfaces of the cupper foil have turned into to form (111) plane and grain boundaries in cupper foil have virtually disappeared, which is different from typical pretreatment using $3 \% \mathrm{H}_{2} / \mathrm{Ar}$ gas as shown in Figure 2.

Figure 3 shows a typical SEM image of graphene grown on the $\mathrm{Cu}$ foil, where hexagonal contrasts due to single-domain graphene are clearly seen. $\mathrm{H}_{2}$ gas was exchanged to $\mathrm{Ar} / \mathrm{H}_{2} / \mathrm{CH}_{4}$ mixed gases (Ar: $97 \%, \mathrm{H}_{2}: 3 \%, \mathrm{CH}_{4}: 0.0002 \%$ ) to grow graphene and finally it provided large-size orientated mono-layer graphene covered both side of the copper substrate. Raman spectra of the so-prepared graphene show intense G and G' band with an intensity ratio of 1 to 2 , which is consistent with monolayer graphene as shown in Figure S1. The Raman spectra were measured after the transfer of the graphene from $\mathrm{Cu}$ foil to a $\mathrm{SiO}_{2} / \mathrm{Si}$ substrate. Long-time $\mathrm{CVD}$ growth of graphene, 
typically 10 hours, using the pretreated cupper foil as a substrate can provide a seamless single sheet of graphene, where the typical size of a grain is about $100 \mathrm{um}^{2}$.

As shown in Figure 3, the mutual orientation of the hexagonal contrasts is unique, indicating that crystal orientation of the present CVD-grown graphene is unique in the area of interest. This unique orientation of the graphene can arise from an epitaxial growth of graphene onto (111) planes of the $\mathrm{Cu}$ foil. As shown in Figure 2, $\mathrm{Cu}$ foils pretreated using the present method (i.e., the high-temperature pure- $\mathrm{H}_{2}$ annealing) show $\mathrm{Cu}$ (111) surface with a minimal amount of grain boundaries. The present long-time CVD growth with the pretreated $\mathrm{Cu}$ foils leads to the formation of well-stitched large-area graphene, which is advantageous to a high-yield preparation of graphene liquid cells. Figure 4 (a) shows a typical TEM image of the current CVD-grow single-layer graphene, whereas Figure4 (b) exhibits the corresponding electron diffraction pattern, where a hexagonal diffraction spot consistent with a single-layer graphene is clearly seen.

The present preparation of the graphene-liquid cell enables us to investigate TEM structural observations of encapsulated liquids. Figure 5 (a) shows a typical TEM image of a graphene liquid cell using the CVD-grown large-area graphene, where pure water droplet is used as liquid encapsulated between the two single-layers of graphene sheets. The dark contrast region corresponds to the graphene water-sandwiched area with a typical lateral size of $160 \sim 300 \mathrm{~nm}$ (see supplementary information). An estimated average thickness of the sandwiched region is $10-20$ water molecule layers. In terms of EELS measurements (see below), the oxygen peak of droplets is very close to that of bulk water, indicating that the chemical environment around oxygen atoms of the observed nano-droplets is very similar to that of bulk water. Black spots seen in the 
TEM image are due to $\mathrm{Cu}$ nanoparticle residues, which are formed and deposited during the transfer process.

The corresponding electron diffraction pattern shown in Figure 5 (b) exhibits two sets of diffraction patterns with six-fold symmetry, which confirms a successful preparation of the graphene sandwiched structure. The electron diffraction shows diffraction spots originating only from the graphene layers and not from the water droplet, suggesting a liquid state of the droplet. The relative angle of the two hexagonal patterns is about 5 degree, indicating that two graphene sheets with a relative angle of 5 degree stack to form the graphene-liquid cell. Relative angles in graphene liquid cells are found to vary from one sample to another due to random stacking caused by the manual stacking procedure used in the present preparation of graphene liquid cells.

Figure 5 (c) shows typical low magnification TEM image of graphene liquid cell. Approximately 35 small droplets are counted in each hole of SiN membrane and near $80 \%$ holes are suspended by bilayer graphene and the graphene liquid cells.

To confirm the presence of water droplet in the sandwiched structure, we performed electron energy loss spectroscopy (EELS) measurements for the sandwiched region. Figure 6 shows an EELS spectrum corresponding to the water sandwiched region shown in Figure4 (a). The observed EELS peaks at 284 and $540 \mathrm{eV}$ originate from the core-loss of carbon and oxygen, respectively. The existence of the EELS peak at $540 \mathrm{eV}$ clearly illustrates the successful encapsulation of water molecules between the graphene layers.

Figure 7 shows a time series of magnified TEM images of a sandwiched water droplet at room temperature. Triangular dark contrasts shown in the Figure correspond to the water droplet sandwiched between two sheets of graphene. Electron diffraction shows 
diffraction spots originating only from the graphene layers which is consistent with the liquid state of the encapsulated water. Importantly, the contrast in the water-sandwiched region decreases as time passes due to the increasing extent of decomposition of the water droplet (due to electron beam induced radiolysis) in a graphene pocket. As seen in these TEM images, a bubble corresponding to the circular low contrast area, which presumably is generated by electron beam irradiation, is observed in the sandwiched region (cf. Supplemental movie 1 and 2). At $252 \mathrm{~s}$, a second (smaller) bubble is appearing in the sandwiched region. These bubbles are frequently observed in water-sandwiched regions during the course of TEM observations. The TEM observation shown here demonstrates that a time-dependent dynamics of liquid water droplets can be observed at nanometer scale using the graphene-sandwiched structure.

\section{Conclusion}

Using the TEM and EELS analysis, the present experiments confirm the encapsulation of water nano-droplets between two single-layer graphene sheets. Typically, small sandwiched droplets as small as $100-300 \mathrm{~nm}$ in size are observed by TEM. In our preparation, we found the average counts of small droplets is 10.2 per um ${ }^{2}$ and we confirmed that one TEM grid have approximately 224000 pockets in our preparation. Direct transferring with spraying the sample mist onto high quality and large size graphene provides high yield of graphene liquid cells. The time-dependent TEM observation often shows a creation of bubbles within the water sandwiched area due to the radiolysis by electron beam irradiation during the observation, which is concomitant with decomposition of the droplets in a graphene pocket. 


\section{Acknowledgments}

This work was partially supported by a Grant-in-Aid for Scientific Research S (No. 22225001 to H.S.), the Grant-in-aid for Young Scientists A (Nos. 25708002 and 24681031 to R.K.), Scientific Research on Innovative Areas (No. 25107002 to R.K.), and the Program for Leading Graduate Schools" Integrative Graduate Education and Research in Green Natural Sciences" of MEXT, Japan. Y.S. thanks JSPS for fellowship. Funding was also provided by the Director, Office of Energy Research, Materials Sciences and Engineering Division, of the US Department of Energy under grant DE-AC02-05CH11231, within the sp2-bonded Materials Program, which provided for TEM characterization; the US National Science Foundation under grant DMR-1206512 which provided for sample synthesis; and the Defense Threat Reduction Agency under grant HDTRA1-13-1-0035 which provided for postdoctoral support. 


\section{References}

[1] K.-L. Liu, C.-C. Wu, Y.-J. Huang, H.-L. Peng, H.-Y. Chang, P. Chang, L. Hsu, T.-R. Yew, LChip 8 (2008) 1915.

[2] A.T. Petkova, R.D. Leapman, Z. Guo, W.-M. Yau, M.P. Mattson, R. Tycko, Science 307 (2005) 262.

[3] H.G. Heide, J. Cell. Biol. 13 (1962) 147.

[4] D. Parsons, Science 186 (1974) 407.

[5] D. Li, H. Zhou, I. Honma, Nature materials 3 (2004) 65.

[6] R.H. Somani, B.S. Hsiao, A. Nogales, S. Srinivas, A.H. Tsou, I. Sics, F.J. Balta-Calleja, T.A. Ezquerra, Macromolecules 33 (2000) 9385.

[7] J.M. Yuk, H.K. Seo, J.W. Choi, J.Y. Lee, ACS nano 8 (2014) 7478.

[8] U. Bergmann, P. Wernet, P. Glatzel, M. Cavalleri, L. Pettersson, A. Nilsson, S. Cramer, PhRvB 66 (2002) 092107.

[9] R. Rosenberg, P. LaRoe, V. Rehn, J. Stöhr, R. Jaeger, C. Parks, PhRvB 28 (1983) 3026.

[10] N. de Jonge, F.M. Ross, Nature nanotech. 6 (2011) 695.

[11] H. Zheng, R.K. Smith, Y.-w. Jun, C. Kisielowski, U. Dahmen, A.P. Alivisatos, Science 324 (2009) 1309.

[12] E. Boyes, P. Gai, Ultmi 67 (1997) 219.

[13] H. Troiani, M. Miki-Yoshida, G. Camacho-Bragado, M. Marques, A. Rubio, J. Ascencio, M. Jose-Yacaman, Nano Lett. 3 (2003) 751.

[14] T. Tsukuda, Bull. Chem. Soc. Jpn. 85 (2012) 151.

[15] M.J. Sharif, M. Yamauchi, S. Toh, S. Matsumura, S.-i. Noro, K. Kato, M. Takata, T. Tsukuda, Nanoscale 5 (2013) 1489.

[16] J.M. Yuk, Q. Zhou, J. Chang, P. Ercius, A.P. Alivisatos, A. Zettl, ACS nano (2015).

[17] J.M. Yuk, J. Park, P. Ercius, K. Kim, D.J. Hellebusch, M.F. Crommie, J.Y. Lee, A. Zettl, A.P. Alivisatos, Science 336 (2012) 61.

[18] M. Jeong, J.M. Yuk, J.Y. Lee, Chem. Mater. (2015).

[19] P. Batson, N. Dellby, O. Krivanek, Nature 418 (2002) 617.

[20] R. Beacham, A. Mac Raighne, D. Maneuski, V. O'Shea, S. McVitie, D. McGrouther, Journal of Instrumentation 6 (2011) C12052.

[21] T. Sasaki, H. Sawada, F. Hosokawa, Y. Sato, K. Suenaga, Ultmi 145 (2014) 50.

[22] A. Bruma, F. Negreiros, S. Xie, T. Tsukuda, R.L. Johnston, A. Fortunelli, Z.Y. Li, Nanoscale 5 (2013) 9620.

[23] H.-G. Liao, D. Zherebetskyy, H. Xin, C. Czarnik, P. Ercius, H. Elmlund, M. Pan, L.-W. Wang, H. Zheng, Science 345 (2014) 916.

[24] J. Wu, W. Gao, J. Wen, D.J. Miller, P. Lu, J.-M. Zuo, H. Yang, Nano Lett. 15 (2015) 2711. [25] M. Jeong, J.M. Yuk, J.Y. Lee, Chem. Mater. 27 (2015) 3200. 
[26] J.C. Meyer, A.K. Geim, M. Katsnelson, K. Novoselov, T. Booth, S. Roth, Nature 446 (2007) 60.

[27] R. Nair, P. Blake, J. Blake, R. Zan, S. Anissimova, U. Bangert, A. Golovanov, S. Morozov, A. Geim, K. Novoselov, Appl. Phys. Lett. 97 (2010) 153102.

[28] C. Lee, X. Wei, J.W. Kysar, J. Hone, Science 321 (2008) 385.

[29] K. Novoselov, A.K. Geim, S. Morozov, D. Jiang, M. Katsnelson, I. Grigorieva, S. Dubonos, A. Firsov, Nature 438 (2005) 197.

[30] G.-H. Lee, R.C. Cooper, S.J. An, S. Lee, A. van der Zande, N. Petrone, A.G. Hammerberg, C. Lee, B. Crawford, W. Oliver, Science 340 (2013) 1073.

[31] Y. Sasaki, R. Kitaura, Y. Yamamoto, S. Arai, S. Suzuki, Y. Miyata, H. Shinohara, Appl. Phys. Express 5 (2012) 065103.

[32] S. Ding, J.S. Chen, D. Luan, F.Y.C. Boey, S. Madhavi, X.W.D. Lou, Chem. Commun. 47 (2011) 5780.

[33] R. Pasricha, S. Gupta, A.K. Srivastava, Small 5 (2009) 2253.

[34] G. Algara-Siller, O. Lehtinen, F. Wang, R. Nair, U. Kaiser, H. Wu, A. Geim, I. Grigorieva, Nature 519 (2015) 443.

[35] H.E. Lim, Y. Miyata, M. Fujihara, S. Okada, Z. Liu, Arifin, K. Sato, H. Omachi, R. Kitaura, S. Irle, K. Suenaga, H. Shinohara, ACS Nano 9 (2015) 5034.

[36] R.R. Meyer, J. Sloan, R.E. Dunin-Borkowski, A.I. Kirkland, M.C. Novotny, S.R. Bailey, J.L. Hutchison, M.L.H. Green, Science 289 (2000) 1324.

[37] X. Li, W. Cai, J. An, S. Kim, J. Nah, D. Yang, R. Piner, A. Velamakanni, I. Jung, E. Tutuc, Science 324 (2009) 1312.

[38] Y. Hao, M.S. Bharathi, L. Wang, Y. Liu, H. Chen, S. Nie, X. Wang, H. Chou, C. Tan, B. Fallahazad, H. Ramanarayan, C.W. Magnuson, E. Tutuc, B.I. Yakobson, K.F. McCarty, Y.-W. Zhang, P. Kim, J. Hone, L. Colombo, R.S. Ruoff, Science 342 (2013) 720 .

[39] P. Zhao, A. Kumamoto, S. Kim, X. Chen, B. Hou, S. Chiashi, E. Einarsson, Y. Ikuhara, S. Maruyama, J. Phys. Chem. C 117 (2013) 10755.

[40] G.H. Han, F. Günes, J.J. Bae, E.S. Kim, S.J. Chae, H.-J. Shin, J.-Y. Choi, D. Pribat, Y.H. Lee, Nano Lett. 11 (2011) 4144.

[41] J. Moser, A. Barreiro, A. Bachtold, Appl. Phys. Lett. 91 (2007) 163513.

[42] Y. Ogawa, B. Hu, C.M. Orofeo, M. Tsuji, K.-i. Ikeda, S. Mizuno, H. Hibino, H. Ago, J. Phys. Chem. Lett. 3 (2012) 219.

[43] S. Ikeda, K. Saiki, Y. Wada, K. Inaba, Y. Ito, H. Kikuchi, K. Terashima, T. Shimada, J. Appl. Phys. 103 (2008) 084313. 


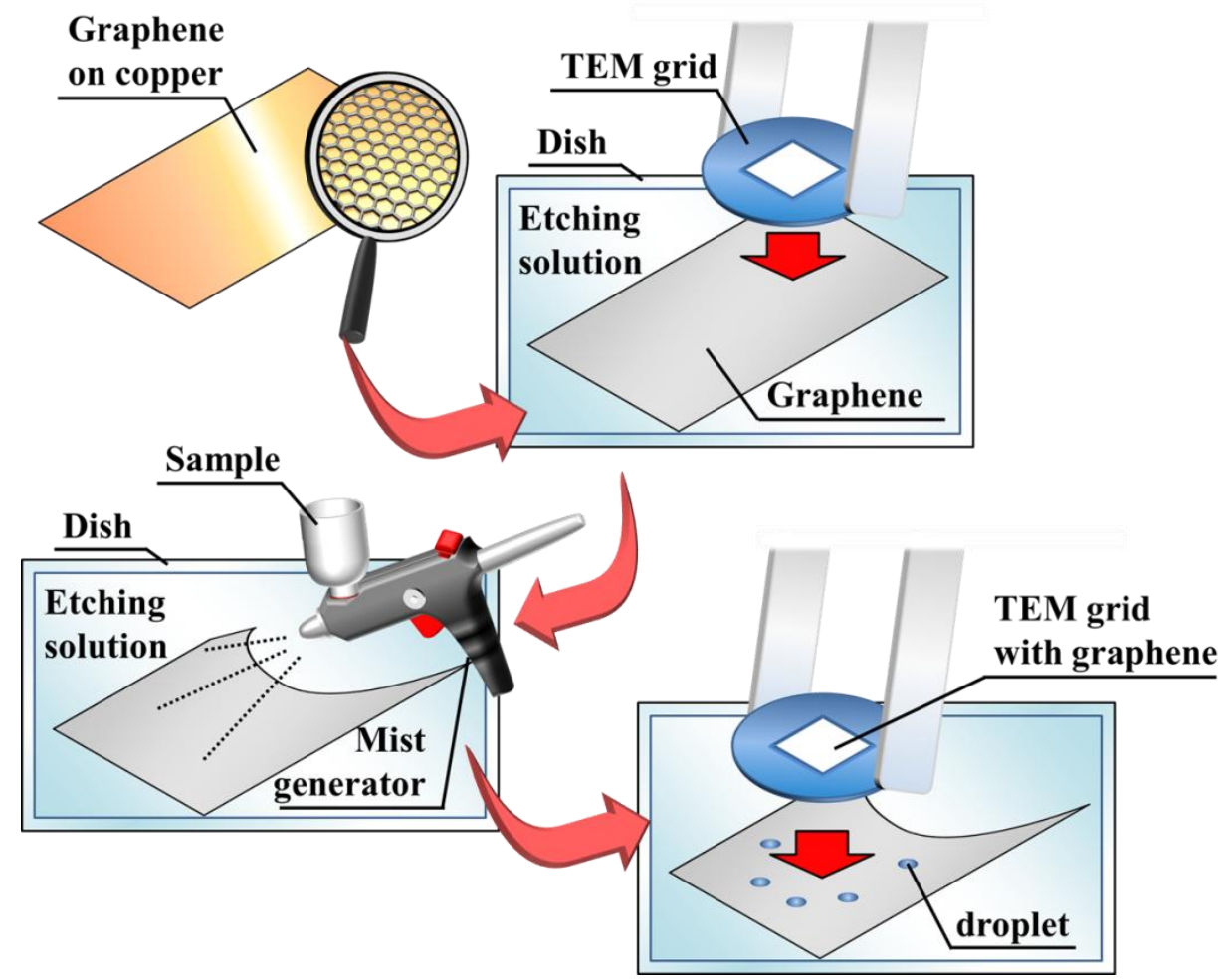

Figure 1. Preparation scheme of the graphene sandwiched structure. Graphene on the solution can be observed by careful irradiation of proper fluorescent light.
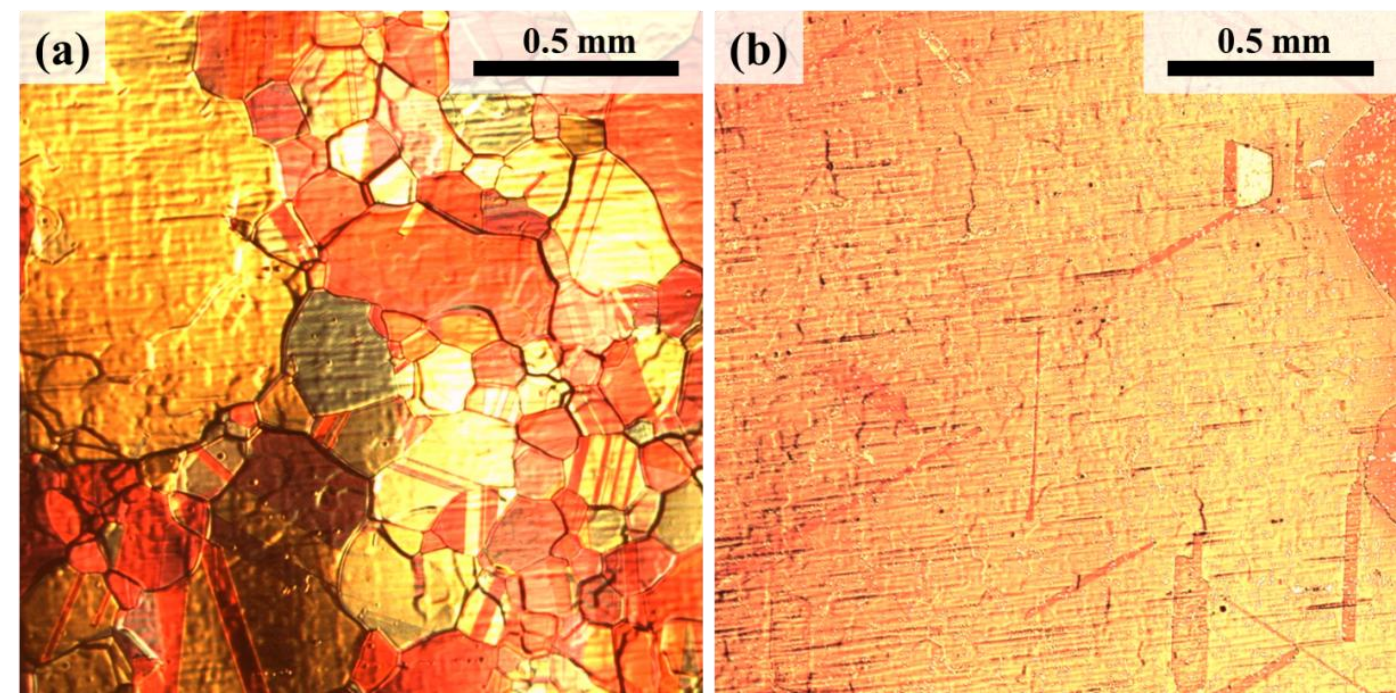

Figure 2. Optical images of copper surface after pretreatment with $\mathrm{Ar} / \mathrm{H}_{2}$ (a) and pure 


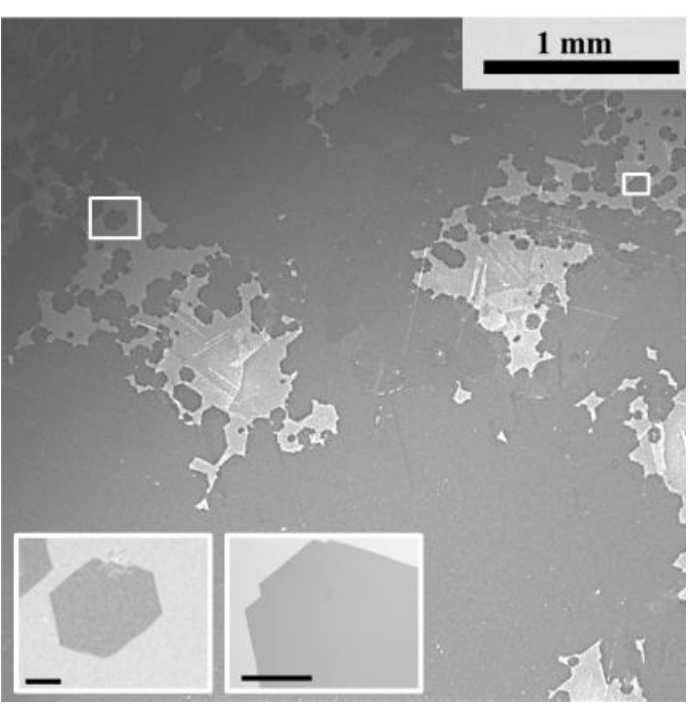

Figure 3. Typical SEM images of single-layer graphene (the dark area) grown on $\mathrm{Cu}$ foil annealed in pure $\mathrm{H}_{2}$ gas. Insert images are zoomed images of the two (white boxed) regions separated about $2 \mathrm{~mm}$. The graphene edges of two images are perpendicular to each other, suggesting that the large area of graphene synthesized maintains high-quality with less grain boundaries. Scale bars in the two insert images are $50 \mathrm{~nm}$. 

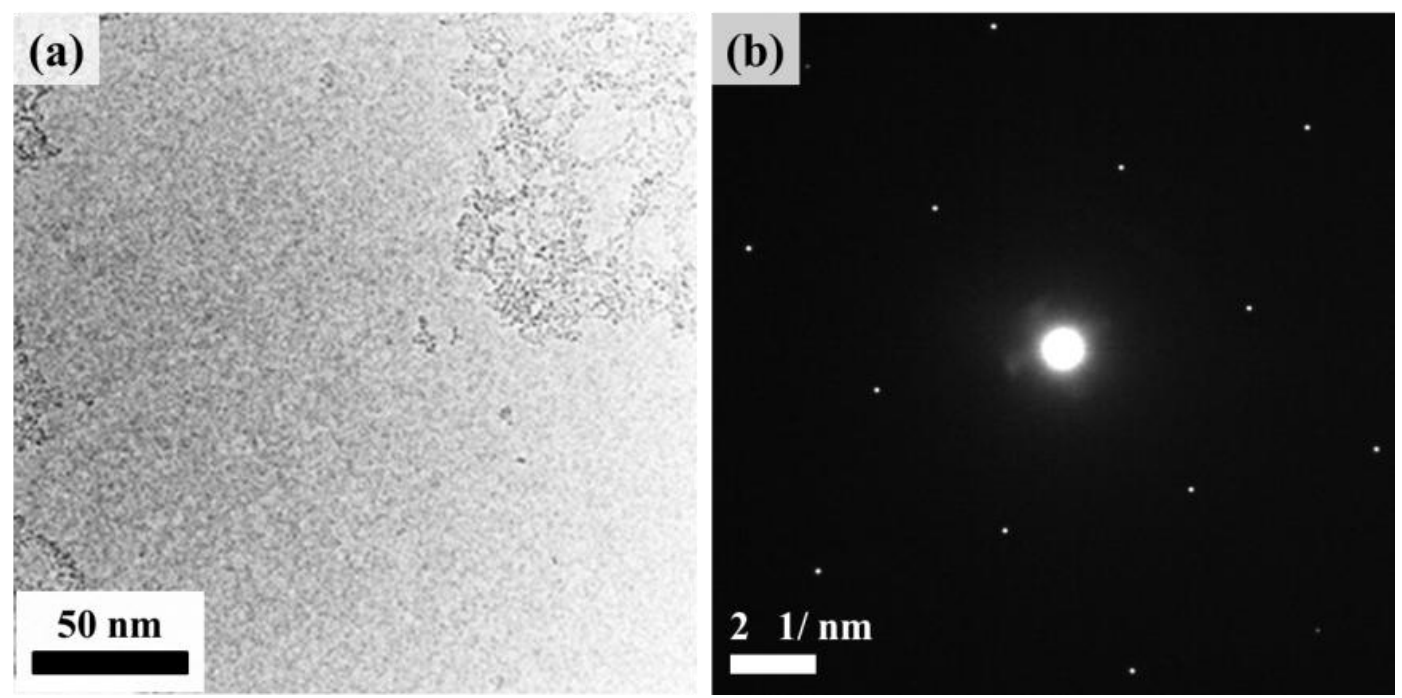

Figure 4. (a) Typical TEM image of the present CVD-grown single-layer graphene. The surfaces are relatively clean except for some amorphous carbon residue (top right) which is attached during the transfer process; (b) the electron diffraction pattern of graphene in Fig. (a). A hexagonal pattern indicates the presence of single-layer graphene.

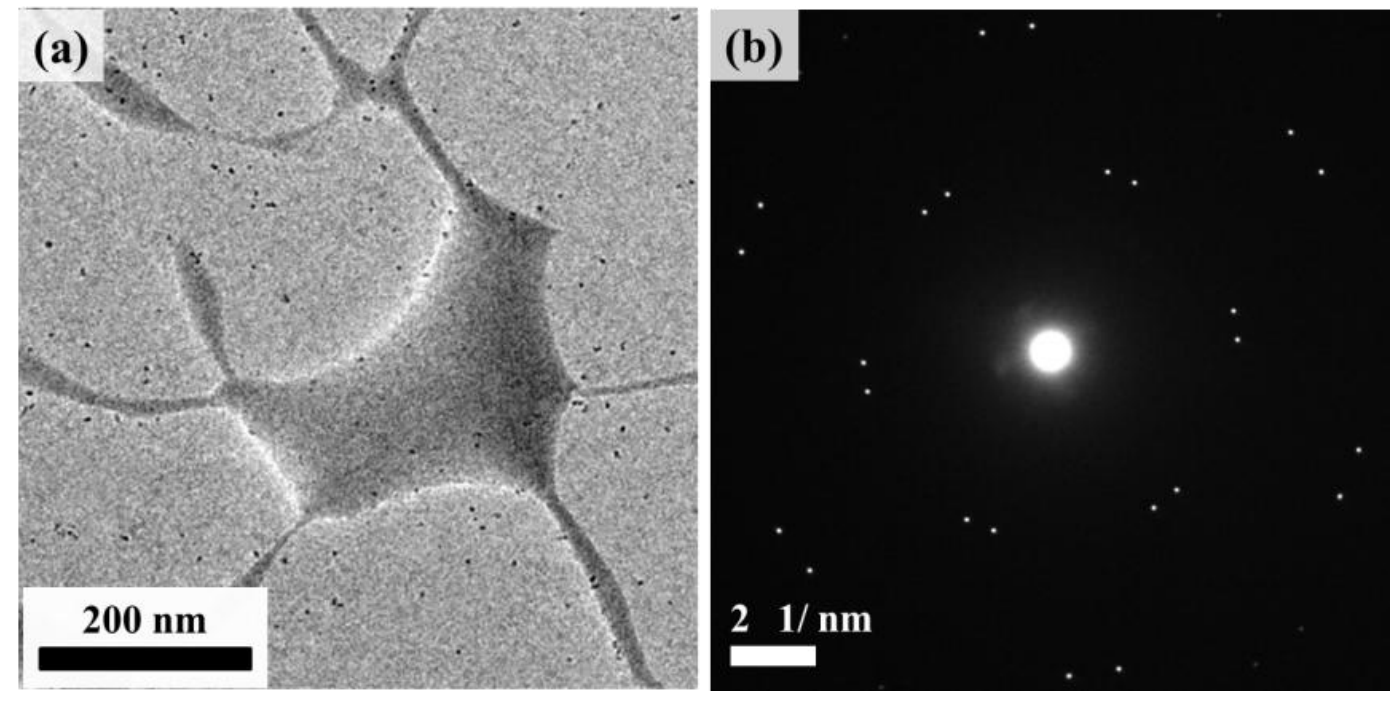




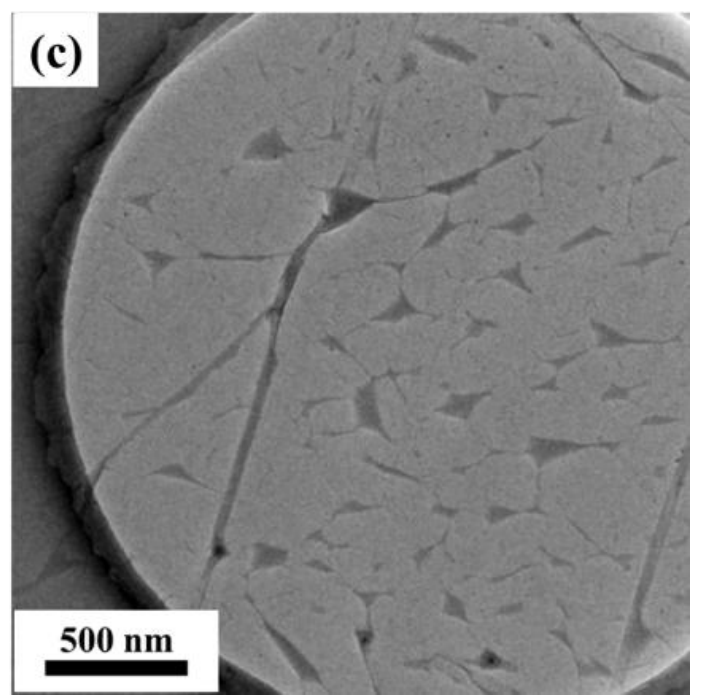

Figure 5. (a) A room temperature typical TEM image of a water-sandwiched area (dark contrast region) by two sheets of single-layer graphene made of Fig.4. Black spots are $\mathrm{Cu}$ nanoparticle residues remained, which are formed and deposited during the transfer process; (b) the electron diffraction pattern of graphene water-sandwiched area in Fig. 4 (a). A twin hexagonal patterns shows the graphene sandwiched structure which is made of two sheets of single-layer graphene. (c) Low magnification typical TEM image of the graphene liquid cell. The SiN membrane, which has 2 um holes, is found in the edge of Fig. 5(c). Many trigonal dark points shown water-encapsulated area is observed in a hole of membrane. 


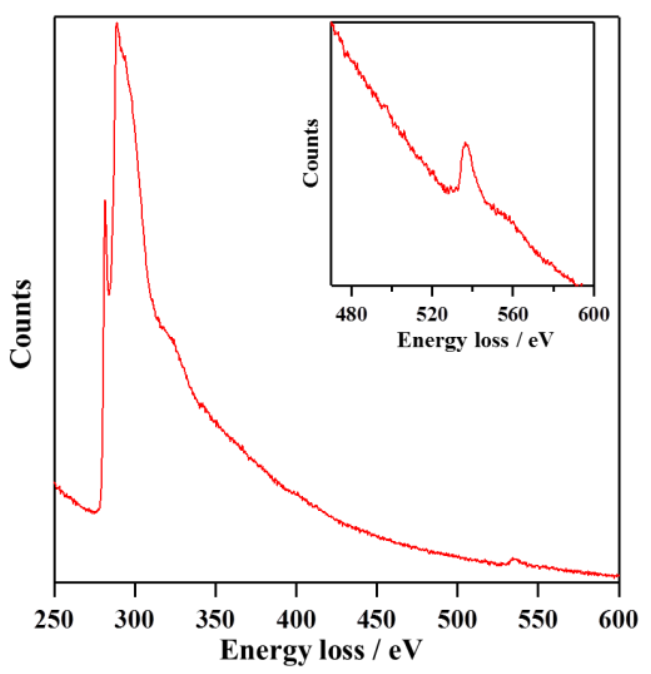

Figure 6. Electron-energy-loss (EELS) spectrum with a selector aperture acquired for the graphene water-sandwiched area in Fig.4. Oxygen K-edge peak $(540 \mathrm{eV})$ due to the sandwiched water droplet is seen together with carbon K-edge peak $(284 \mathrm{eV})$. The inset shows an expanded view of oxygen peak area. 


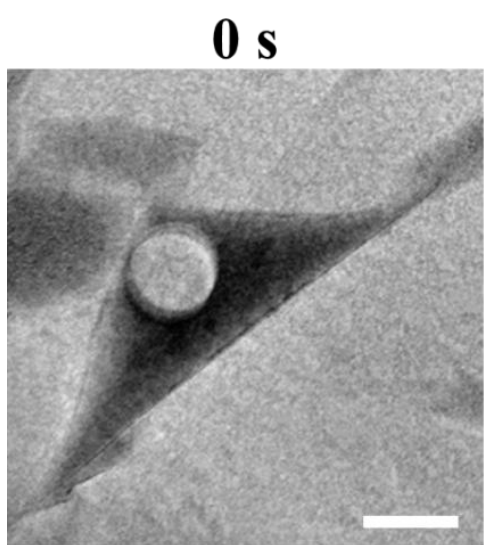

$66 s$
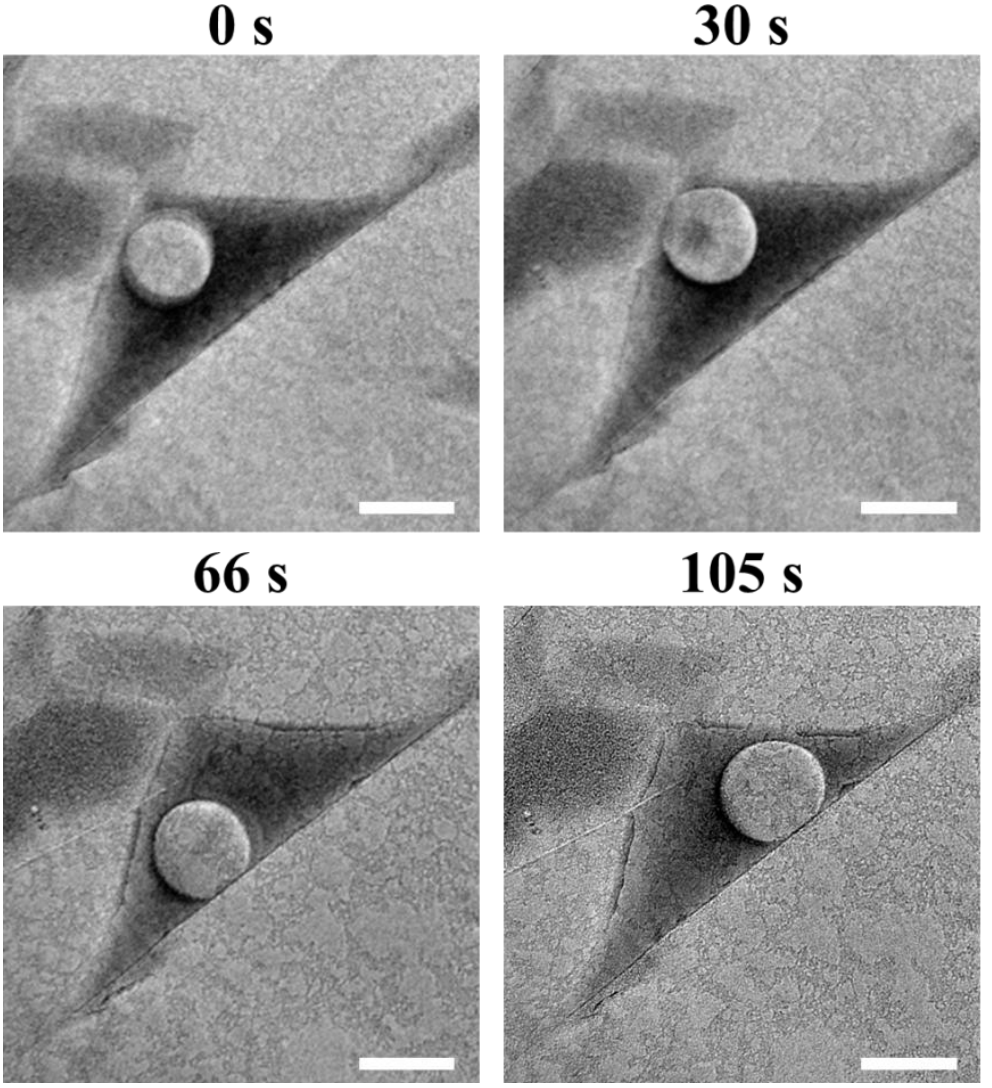

$105 \mathrm{~s}$
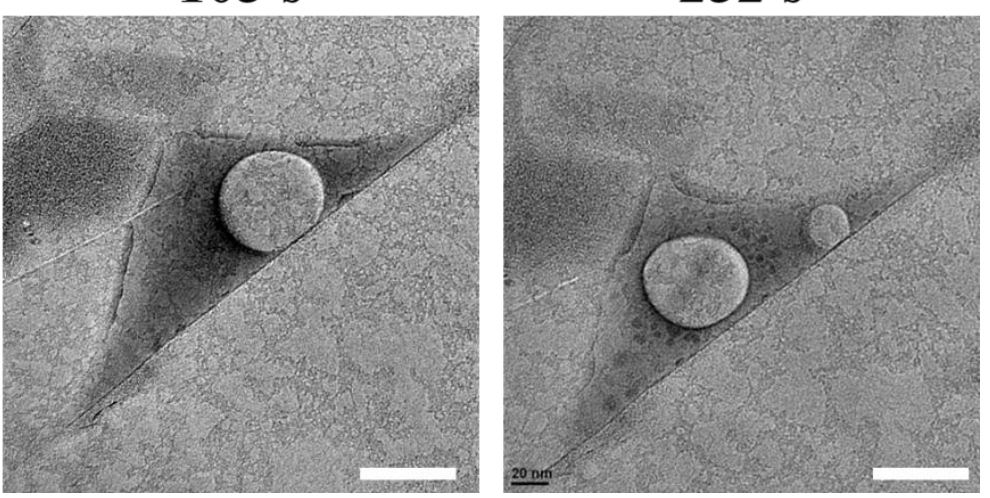

Figure 7. Time series of room temperature TEM images of graphene water-sandwiched region (triangle dark contrast area) at room temperature with a scale bar of $50 \mathrm{~nm}$. A bubble (circular low contrast area), which stems from electron beam irradiation, is also seen in the sandwiched region. The contrast in the water-sandwiched region decreases as time passes due to the increasing decomposition of the water droplet from the interstitial space of the two single-layer graphene. At $252 \mathrm{~s}$, a second (smaller) bubble is appearing in the sandwiched region. 